\title{
CARACTERIZAÇÃO QUÍMICA E ATIVIDADE MICROBIANA DE COPRÓLITOS DE Chibui bari (Oligochaeta) E DO SOLO ADJACENTE ${ }^{(1)}$
}

\author{
Sergio da Silva Fiuza ${ }^{(2)}$, Jorge Ferreira Kusdra ${ }^{(3)} \&$ Denise Temporim Furtado $^{(4)}$
}

\section{RESUMO}

Chibui bari é um oligochaeta edáfico nativo da região amazônica, considerado como minhocuçu em razão do seu grande tamanho (até $60 \mathrm{~cm}$ de comprimento). Seus excrementos ou coprólitos são liberados em montículos com formato de torre, com altura de até $30 \mathrm{~cm}$ e com matéria seca de até $2,0 \mathrm{~kg}$. $O$ objetivo desta pesquisa foi comparar a disponibilidade de nutrientes, o teor de $\mathrm{C}$ orgânico e a atividade microbiana dos coprólitos de Chibui bari com o solo adjacente de áreas de floresta secundária, seringal de cultivo e pastagem, localizadas na Universidade Federal do Acre - UFAC, em Rio Branco, Acre. As coletas foram realizadas considerando o delineamento em blocos casualizados, com três tratamentos, constituídos por: a) coprólitos de Chibui bari e por amostras compostas de solos retiradas no raio de $10 \mathrm{~cm}$ de cada coprólito coletado, nas profundidades de b) 0-10 cm e c) 10-20 cm. Os resultados foram submetidos à análise de variância, e as médias dos tratamentos, comparadas pelo teste de Tukey a $5 \%$. Além disso, efetuou-se análise de correlação simples entre as variáveis. Os coprólitos apresentaram maiores valores de $\mathrm{pH}, \mathrm{P}$ disponível, bases trocáveis $\left(\mathrm{Ca}^{2+}, \mathrm{Mg}^{2+}, \mathrm{K}^{+}\right), \mathrm{C}$ orgânico e atividade microbiana. $\mathrm{O}$ teor de $\mathrm{Al}^{3+}$ foi menor nos coprólitos do que no solo na camada de 0-20 cm em $55 \%$ (pastagem), $62 \%$ (seringal) e $70 \%$ (floresta). Verificaram-se, ainda, correlações

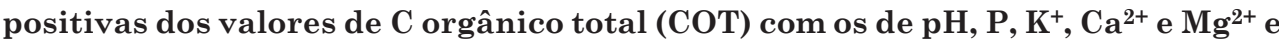
correlações negativas de $\mathrm{Al}^{3+}$ com $\mathrm{COT}, \mathrm{pH}, \mathrm{P}, \mathrm{K}^{+}, \mathrm{Ca}^{2+}$ e $\mathrm{Mg}^{2+}$ nas três áreas avaliadas.

Termos de indexação: minhocuçu, fertilidade do solo, excrementos de minhocas.

\footnotetext{
(1) Parte da Dissertação de Mestrado do primeiro autor apresentada ao Curso de Pós-graduação em Agronomia - Produção Vegetal da Universidade Federal do Acre - UFAC. Bolsista da CAPES. Financiada com recursos do Fundo de Desenvolvimento Científico e Tecnológico do Estado do Acre - FDCT. Recebido para publicação em março de 2010 e aprovado em janeiro de 2011.

${ }^{(2)}$ Engenheiro-Agrônomo, Mestre em Agronomia, Universidade Federal do Acre - UFAC. CEP 69915-900 Rio Branco (AC). Email: ssfiuza@globo.com

(3) Professor Adjunto do Centro de Ciências Biológicas e da Natureza, UFAC. E-mail: kusdra@globo.com

(4) Engenheira-Agrônoma, Mestranda em Agronomia, UFAC. Bolsista da CAPES. E-mail: denisetemporim@globo.com
} 


\title{
SUMMARY: CHEMICAL PROPERTIES AND MICROBIAL ACTIVITY IN CASTINGS OF Chibui bari (Oligochaeta) AND SURROUNDING SOIL
}

\begin{abstract}
Chibui bari is an edaphic oligochaeta from the Amazon region, called "minhocuçu" in view of its large size (length up to $60 \mathrm{~cm}$ ). Their castings, in tower-shaped mounds with a height of up to $30 \mathrm{~cm}$ and dry weight of $2 \mathrm{~kg}$, are common on the soil surface of Acre State. The objective of this study was to compare nutrient availability, organic carbon concentration and microbial activity in Chibui bari castings with soil of the surroundings under secondary forest, rubber cultivation and pasture, in areas of the Federal University of Acre - UFAC in Rio Branco, Acre. The experiment was arranged in a randomized block design, with three treatments consisting of a) Chibui bari castings and soil samples from within a radius of $10 \mathrm{~cm}$ from each casting from depths of b) $0-10 \mathrm{~cm}$ and c) 10-20 cm. The results were submitted to analysis of variance and treatment means compared by the Tukey test at $5 \%$ probability. In addition, the simple correlation between the variables was analyzed. The $p H$ values, available $P$, exchangeable cations $\left(\mathrm{Ca}^{2+}, \mathrm{Mg}^{2+}, \mathrm{K}^{+}\right)$, organic carbon and microbial activity were higher in the castings than in the surrounding soil, whereas the $\mathrm{Al}^{3+}$ concentration was lower in castings in the 0 $20 \mathrm{~cm}$ layer by $55 \%$ (pasture), $62 \%$ (rubber cultivation) and $70 \%$ (forest). There was also a positive correlation of soil organic carbon values with $\mathrm{pH}, \mathrm{P}, \mathrm{K}^{+}, \mathrm{Ca}^{2+}$ and $\mathrm{Mg}^{2+}$ and negative correlations of $\mathrm{Al}^{3+}$ with soil organic carbon, $\mathrm{pH}, \mathrm{K}^{+}, \mathrm{Ca}^{2+}$ and $\mathrm{Mg}^{2+}$ in the three areas.
\end{abstract}

Index terms: Minhocuçu, earthworm castings, soil fertility.

\section{INTRODUÇÃO}

As minhocas, junto com formigas e cupins, são consideradas os mais importantes engenheiros do solo (Jouquet et al., 2006), pois são organismos que direta ou indiretamente regulam a disponibilidade de recursos a outras espécies (Jones et al., 1994). As galerias e os coprólitos desses animais são definidos como estruturas biogênicas (Rossi et al., 2006) que causam alterações nas propriedades físicas, químicas e biológicas do solo (Lafont, 2007), sendo importantes na formação e manutenção da fertilidade em agroecossistemas (Hendrix, 1995).

Brito-Vega \& Espinosa-Victoria (2009) encontraram, no trato digestório de minhocas anécicas, epigeicas e endogeicas, microrganismos envolvidos em importantes processos no solo, destacando-se fixadores de N, produtores de hormônios de crescimento vegetal e solubilizadores de fosfato. De acordo com esses autores, as minhocas são incubadoras e dispersoras desses microrganismos.

Devido à ingestão de materiais minerais e orgânicos pelas minhocas geófagas, seus coprólitos podem conter maiores quantidades de $\mathrm{C}$ orgânico, $\mathrm{N}$ total e $\mathrm{P}$ inorgânico do que o solo. Os recursos adicionados ao material ingerido pelas minhocas permanecem nos excrementos, aumentando as taxas de mineralização de nutrientes por curto período de tempo, até que os coprólitos comecem a secar e, então, a decomposição da matéria orgânica, a mineralização de nutrientes e a atividade microbiana tende a estabilizar, atingindo, muitas vezes, níveis inferiores aos observados no solo não ingerido (Brown et al., 2000). Essa sequência de eventos é importante para a regulação da matéria orgânica, para o estoque de $\mathrm{C}$ no solo e para a formação de macro e microagregados (Logsdon \& Linden, 1992; Pulleman et al., 2004; Blouin et al., 2006; Lavelle et al., 2006).

Chibui bari (Glossoscolecidae, oligochaeta) é um minhocuçu geófago e endogeico com distribuição em todo o Estado do Acre que demonstra facilidade de adaptação em sistemas de pastagem e cultivos perenes, apresentando preferência por áreas antrópicas (Guerra, 1988a). Esses animais produzem grande quantidade de coprólitos durante seis a oito meses do ano, que correspondem ao período de alta precipitação pluvial e umidade do solo, favoráveis à atividade da espécie. A produção anual de matéria seca de coprólitos em habitat de Chibui bari no Acre foi avaliada entre 9 e 143 t ha-1 $^{-1}$ Guerra, 1988a; Fiuza, 2009). Os coprólitos constituem montículos em formato de torre, depositados na superfície de vários solos da região amazônica, sendo estes diariamente aumentados com adição de excrementos semilíquidos na sua parte superior. Em condições naturais, as torres de coprólitos, depois de secas, resistem por período de meses a anos, quando então se fragmentam em blocos globulares, que são incorporados à superfície do solo.

Em razão do baixo valor energético do alimento que é predominantemente mineral, as espécies endogeicas necessitam ingerir grande quantidade desse material para atender às suas exigências energéticas (Lee, 1985), o que resulta em alta produção de excrementos que são liberados na superfície e 
subsuperfície do solo. De acordo com Lavelle et al. (2004), as espécies de minhocas endogeicas depositam coprólitos contendo proporção significativa de matéria orgânica.

Coprólitos de Chibui bari foram utilizados na composição de substratos para produção de mudas de mamoeiro, alface e couve-manteiga (Silva et al., 2007b; Kusdra et al., 2008; Souza et al., 2008). A caracterização química realizada nos substratos contendo coprólitos e solo distrófico mostrou que nos primeiros havia maior teor de $\mathrm{Ca}^{2+}, \mathrm{Mg}^{2+}$, $\mathrm{P}$ e matéria orgânica, além de estes possuírem $\mathrm{pH}$ mais elevado e menor teor de $\mathrm{Al}^{3+}$. Entretanto, são necessários ainda vários trabalhos visando avaliar o potencial agronômico dessa espécie, principalmente no sentido de dimensionar a magnitude de sua contribuição para solo e plantas.

O objetivo desta pesquisa foi comparar a disponibilidade de nutrientes, o teor de $\mathrm{C}$ orgânico e a atividade microbiana dos coprólitos com o solo adjacente em áreas de floresta secundária, seringal de cultivo e pastagem.

\section{MATERIAL E MÉTODOS}

Foram realizados experimentos distintos em áreas de floresta secundária, seringal de cultivo e pastagem, localizadas na Universidade Federal do Acre - UFAC, na cidade de Rio Branco, Acre. A pastagem de 1,5 ha tem cobertura de capim Brachiaria spp., com pastejo eventual de animais. O seringal de cultivo (Hevea brasiliensis), com 20 anos, encontra-se numa área de 2,0 ha. A floresta secundária está inserida no Parque Zoobotânico da UFAC, que possui área total de 100 ha.

As três áreas citadas foram selecionadas para realização das coletas por serem habitat de Chibui bari (Glossoscolecidae, oligochaeta), onde não há realização de captura desses minhocuçus.

Os solos das áreas estudadas são classificados, de acordo com o Sistema Brasileiro de Classificação de Solos (Embrapa, 2006), como Argissolo VermelhoAmarelo alítico plíntico.

As coletas dos coprólitos e dos solos foram realizadas no delineamento em blocos casualizados, considerando três tratamentos, em três blocos, com cinco repetições por bloco. Os blocos foram estabelecidos com as dimensões de 14 x $40 \mathrm{~m}$, em área total de $1.680 \mathrm{~m}^{2}$, em cada uma das três áreas descritas.

Os tratamentos foram constituídos pelos coprólitos de Chibui bari (tratamento 1) e por amostras compostas de solos das profundidades de $0-10 \mathrm{~cm}$ (tratamento 2) e 10-20 cm (tratamento 3), totalizando 45 amostras por área estudada. A amostragem de solo foi feita utilizando-se trado calador em cinco pontos adjacentes a $10 \mathrm{~cm}$ de raio de cada coprólito coletado, obtendo-se uma amostra composta.

Os coprólitos foram coletados manual e individualmente, selecionando-se aleatoriamente no interior dos blocos aqueles com formato de torre preservado, evitando-se os fragmentados e misturados com serapilheira ou com terra da camada superficial.

As análises químicas do solo e dos coprólitos foram realizadas conforme Embrapa (1997), sendo o $\mathrm{pH}$ determinado em $\mathrm{H}_{2} \mathrm{O}$ na proporção solo:água de 1:2,5; $\mathrm{Al}^{3+}, \mathrm{Ca}^{2+} \mathrm{e} \mathrm{Mg}^{2+}$ foram extraídos com solução de $\mathrm{KCl}$ $1 \mathrm{~mol} \mathrm{~L}^{-1}$, na proporção solo:solução de 1:10; $\mathrm{K}^{+}$e P foram extraídos com solução de $\mathrm{HCl} 0,05 \mathrm{~mol} \mathrm{~L}^{-1} \mathrm{e}$ $\mathrm{H}_{2} \mathrm{SO}_{4} 0,0125 \mathrm{~mol} \mathrm{~L}^{-1}$, na proporção solo:solução de 1:10, em que o $\mathrm{K}$ foi determinado por fotometria de chama, o P, por colorimetria, e o C orgânico total (COT), por oxidação da matéria orgânica com dicromato de potássio $\left(\mathrm{K}_{2} \mathrm{Cr}_{2} \mathrm{O}_{7}\right) 0,2 \mathrm{~mol} \mathrm{~L}^{-1} \mathrm{em}$ meio sulfúrico e titulado com sulfato ferroso amoniacal $0,1 \mathrm{~mol} \mathrm{~L}^{-1} ; \mathrm{e} \mathrm{H}+\mathrm{Al}$ foram extraídos com solução de acetato de cálcio $1 \mathrm{~mol} \mathrm{~L}^{-1}$, ajustada a $\mathrm{pH} 7$, na proporção de 1:15. A partir dos resultados, foram calculadas a soma de bases trocáveis (SB), a capacidade efetiva de troca catiônica (CTC), a porcentagem de saturação por bases trocáveis $(\mathrm{V} \%)$ e a saturação por $\mathrm{Al}(\mathrm{m})$. A respiração microbiana do solo (RMS) foi determinada de acordo com Silva et al. (2007a).

Os resultados dos experimentos foram submetidos à verificação de normalidade dos resíduos pelo teste de Shapiro-Wilk, homogeneidade de variâncias, pelos testes de Bartlett ou Hartley, e análise de variância (teste F), sendo as médias dos tratamentos comparadas pelo teste de Tukey a $5 \%$. Além disso, efetuou-se análise de correlação linear simples entre os resultados das variáveis químicas.

\section{RESULTADOS E DISCUSSÃO}

Considerando os coprólitos da área de floresta secundária, as médias (Quadro 1) foram maiores em relação ao solo de $0-10 \mathrm{~cm}$ na seguinte magnitude: $P$, $96 \%$; $\mathrm{K}^{+}, 33 \%$; $\mathrm{Ca}^{2+}, 222 \%$; $\mathrm{Mg}^{2+}, 98 \%$; COT, $86 \%$; RMS, $358 \%$; e em relação ao solo de $10-20 \mathrm{~cm}$, as médias foram superiores em: $\mathrm{P}, 321 \%$; $\mathrm{K}^{+}, 100 \%$; $\mathrm{Ca}^{2+}, 473 \%$; $\mathrm{Mg}^{2+}, 234 \%$; COT, $248 \%$; e RMS, $769 \%$. $\mathrm{O} \mathrm{pH}$ nos coprólitos foi significativamente maior $(\mathrm{p}<0,05)$ que o do solo nas três áreas pesquisadas (Quadros 1,2 e 3), sendo, contudo, baixa a magnitude de incremento.

Considerando os coprólitos, as médias dos resultados da área de pastagem (Quadro 1) foram maiores em relação ao solo de $0-10 \mathrm{~cm}$ na seguinte magnitude: $\mathrm{P}, 58 \%$; $\mathrm{K}^{+}, 45 \%$; $\mathrm{Ca}^{2+}, 70 \%$; $\mathrm{Mg}^{2+}$, $50 \%$; COT, $42 \%$; RMS, 97 \%; e em relação ao solo de 10-20 cm, as médias foram superiores em: P, $291 \%$; 
Quadro 1. Resultados das análises químicas e atividade microbiana dos coprólitos e do solo adjacente nas profundidades de 0-10 e 10-20 cm, em área de floresta secundária, pastagem e seringal de cultivo

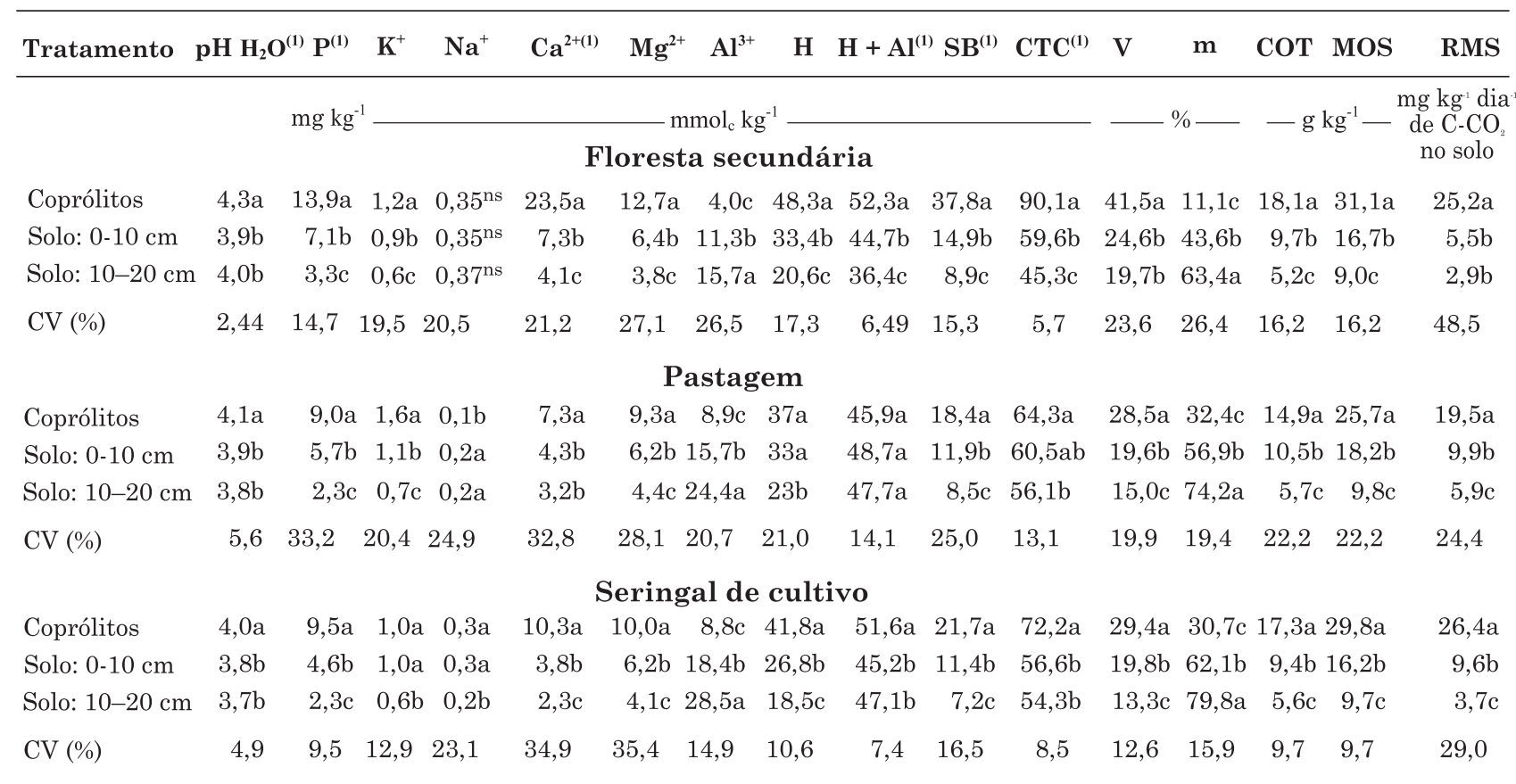

(1) Dados originais que foram transformados em para a variável $\mathrm{H}+\mathrm{Al}$ a fim de atender aos pressupostos da análise de variância (normalidade dos resíduos e,ou, homogeneidade de variâncias). Médias seguidas pela mesma letra, na coluna, não diferem entre si pelo teste de Tukey ( $>$ > 0,05). Soma de bases (SB); capacidade de troca de cátions (CTC); saturação por bases (V); saturação por alumínio (m); C orgânico total (COT); matéria orgânica do solo (MOS); respiração microbiana do solo (RMS).

$\mathrm{K}^{+}, 128 \% ; \mathrm{Ca}^{2+}, 128 \% ; \mathrm{Mg}^{2+}, 111 \% ; \mathrm{COT}, 161 \% ; \mathrm{e}$ RMS, $230 \%$.

Considerando os coprólitos da área de seringal de cultivo, as médias (Quadro 1) foram maiores em relação ao solo de $0-10 \mathrm{~cm}$, na seguinte magnitude: $\mathrm{P}, 106 \%$; $\mathrm{Ca}^{2+}, 171 \%$; $\mathrm{Mg}^{2+}, 61 \%$; COT, $84 \%$, RMS, $175 \%$; e em relação ao solo de $10-20 \mathrm{~cm}$, as médias foram superiores em: $\mathrm{P}, 313 \%$, $\mathrm{K}^{+}, 67 \%$; $\mathrm{Ca}^{2+}, 347 \%$; $\mathrm{Mg}^{2+}$, $144 \%$; COT, 209 \%; e RMS, $613 \%$.

Correlações positivas foram obtidas entre os resultados de $\mathrm{COT}$ com os de $\mathrm{pH}, \mathrm{P}, \mathrm{K}^{+}, \mathrm{Ca}^{2+}, \mathrm{Mg}^{2+}$ dos coprólitos e do solo de 0-10 cm e de 10-20 cm nas três áreas estudadas, ao passo que o teor de $\mathrm{Al}^{3+}$ foi menor $(\mathrm{p}<0,01)$ nos coprólitos que no solo, tendo seus valores apresentado correlação negativa com COT, $\mathrm{pH}$, $\mathrm{Ca}^{2+}, \mathrm{Mg}^{2+}$ e $\mathrm{K}^{+}$(Quadro 2).

Pelas análises de correlação entre as variáveis, verificou-se que a maior qualidade química dos coprólitos é explicada pelo efeito de concentração de COT e pelo aumento da atividade microbiana, que acelera os processos de mineralização, aumentando a saturação por bases e reduzindo os teores de $\mathrm{Al}^{3+}$.

No curso da decomposição, ampla variedade de ácidos orgânicos é liberada ou sintetizada pelos microrganismos decompositores, que podem formar complexos estáveis com o $\mathrm{Al}^{3+}$ (Pavinato \& Rosolem, 2008), além de o aumento da saturação por bases nos coprólitos competir pelos sítios de troca, precipitandoos. A redução do teor de $\mathrm{Al}^{3+}$ associada ao aumento do pH promovida por Chibui bari nos seus coprólitos, em relação ao solo de origem, pode ter efeito positivo no aumento da disponibilidade de $\mathrm{P}$, diminuindo sua precipitação. Pashanasi et al. (1996) também observaram incremento significativo no valor de $\mathrm{pH}$

Quadro 2. Coeficientes de correlação linear (r) do teor de $\mathrm{C}$ orgânico total (COT) e do teor de $\mathrm{Al}^{3+}$ com $\mathrm{pH}, \mathrm{P}, \mathrm{K}^{+}, \mathrm{Ca}^{2+}$ e $\mathrm{Mg}^{2+}$ avaliados nos coprólitos de Chibui bari, no solo de 0-10 e 10$20 \mathrm{~cm}$ de profundidade, nas áreas de floresta, pastagem e seringal

\begin{tabular}{|c|c|c|c|c|c|c|c|}
\hline & Área & $\mathbf{p H}$ & $\mathbf{P}$ & $\mathbf{K}^{+}$ & $\mathrm{Ca}^{2+}$ & $\mathrm{Mg}^{2+}$ & COT \\
\hline \multirow[t]{3}{*}{ COT } & Floresta & $0,50 * *$ & $0,92^{* *}$ & $0,81^{* *}$ & $0,83^{* *}$ & $0,93^{* *}$ & \\
\hline & Pastagem & $0,46^{* *}$ & $0,89^{* *}$ & $0,89^{* *}$ & $0,82^{* *}$ & $0,88^{* *}$ & \\
\hline & Seringal & $0,58 * *$ & $0,91^{* *}$ & $0,70 * *$ & $0,74^{* *}$ & $0,74^{* *}$ & \\
\hline \multirow[t]{3}{*}{$\mathrm{Al}^{3+}$} & Floresta & $-0,67 * *$ & $-0,76^{* *}$ & $-0,78^{* *}$ & $-0,83^{* *}$ & $-0,86^{* *}$ & $-0,86^{* *}$ \\
\hline & Pastagem & $-0,64^{* *}$ & $-0,82^{* *}$ & $-0,85^{* *}$ & $-0,86^{* *}$ & $-0,81^{* *}$ & $-0,88^{* *}$ \\
\hline & Seringal & $-0,63^{* *}$ & $-0,88^{* *}$ & $-0,76^{* *}$ & $-0,74^{* *}$ & $-0,78^{* *}$ & $-0,93^{* *}$ \\
\hline
\end{tabular}

$\left.\overline{\mathrm{P}(\mathrm{mg} \mathrm{kg}}{ }^{-1}\right) ; \mathrm{K}^{+}, \mathrm{Ca}^{2+}, \mathrm{Mg}^{2+} \mathrm{e} \mathrm{Al}^{3+}\left(\mathrm{mmol}_{\mathrm{c}} \mathrm{dm}^{-3}\right) ; \mathrm{COT}\left(\mathrm{g} \mathrm{kg}^{-1}\right)$. **: indicam $\mathrm{p}<0,01$. 
de um solo de floresta onde foram introduzidas Pontoscolex corethrurus.

Segundo Buck et al. (1999), o maior teor de nutrientes nos coprólitos ocorre independentemente de a espécie de minhoca ser epigeica ou endogeica, em diferentes tipos de coberturas do solo e de cultivos agrícolas. Oyedele et al. (2006) verificaram nos coprólitos de Hyperiodrilus africanus significativo enriquecimento em bases trocáveis, matéria orgânica, saturação por bases e CTC. Chaoui et al. (2003) relataram incrementos de $\mathrm{P}$ e de $\mathrm{K}$ e maior respiração microbiana nos excrementos de Lumbricus rubellus, e Quadros et al. (2002), maiores teores de $\mathrm{Ca}^{2+}, \mathrm{Mg}^{2+}$ e $\mathrm{K}^{+}(12,5,10,0$ e 3,0 vezes mais, respectivamente) nos coprólitos que no solo de $0-5 \mathrm{~cm}$ de profundidade em área de cultivo de Eucalyptus grandis.

Vários autores (Souza et al., 2008; Zaia et al., 2008a,b) afirmam que os teores de C orgânico e a atividade microbiana são geralmente maiores em coprólitos do que na camada de $0-5 \mathrm{~cm}$ do solo. Bossuyt et al. (2005) observaram que os agregados biogênicos formados por coprólitos de Aporrectodea caliginosa foram enriquecidos em $22 \%$ de C orgânico, em comparação aos agregados fisicogênicos do solo. Segundo Brown et al. (2000), mesmo habitando camadas subsuperficiais do solo, pobre em matéria orgânica, as espécies geófagas e endogeicas conseguem aumentar a decomposição de formas estáveis de C ingerido. Isso é possível, segundo esses autores, devido ao umedecimento e à adição de muco e de microrganismos mutualistas do sistema digestório, que promovem o aumento das taxas de mineralização nos coprólitos, podendo estes, de acordo com James \& Brown (2008), constituir-se em importantes fontes de nutrientes para as plantas.

Os teores de P disponível no solo de 0-10 e 10$20 \mathrm{~cm}$ nas três áreas podem ser considerados baixos. No entanto, nos coprólitos de Chibui bari, os valores podem ser interpretados como médios a altos, de acordo com as classes de disponibilidade de $\mathrm{P}$ em função do teor de argila (Malavolta, 2006). O P orgânico pode representar de 15 a $80 \%$ do P total do solo (Dechen \& Nachtigall, 2007), e sua liberação depende da atividade microbiana. Chapuis-Lardy et al. (2009) estudaram o efeito da ingestão do solo por minhocas geófagas endogeicas (Pontoscolex corethrurus) na disponibilidade do P. Estes autores relataram que o teor de $\mathrm{P}$ disponível aumentou 116 \% nos coprólitos em relação ao solo a partir do qual foram derivados. Contudo, destacaram que somente a presença de minhocas não foi capaz de eliminar problemas de deficiência desse nutriente.

Em áreas de cultivos permanentes e florestas, a produção de coprólitos pode estar integrada à estratégia de manutenção do equilíbrio dinâmico da ciclagem, uma vez que esses animais fazem remobilização para a superfície do solo de compostos orgânicos e de nutrientes perdidos para a subsuperfície.
O fato de se ter verificado que os coprólitos apresentaram melhor condição química e biológica do que o solo adjacente nas três áreas avaliadas aponta para a importância do manejo conservacionista e indica a possibilidade de aumento no crescimento de plantas e da produtividade das culturas instaladas em áreas agrícolas onde há produção de coprólitos de Chibui bari, desde que as práticas de manejo do solo e da cultura não tragam prejuízo à sobrevivência dos animais.

\section{CONCLUSÕES}

1. Os coprólitos de Chibui bari, comparados ao solo adjacente, concentram nutrientes $\left(\mathrm{Ca}^{2+}, \mathrm{Mg}^{2+}, \mathrm{K}^{+}, \mathrm{P}\right)$ e COT, aumentam a respiração microbiana, a CTC e o $\mathrm{pH}$ e reduzem os teores de $\mathrm{Al}^{3+}$.

2. As maiores atividade microbiana e quantidade de COT nos coprólitos de Chibui bari aumentam a mineralização e a disponibilidade de nutrientes.

\section{AGRADECIMENTOS}

À Coordenação de Aperfeiçoamento de Pessoal de Nível Superior - CAPES, pela concessão de bolsa de estudos, e à Fundação de Tecnologia do Estado do Acre - FUNTAC, pelo apoio financeiro do Fundo de Desenvolvimento Científico e Tecnológico - FDCT no âmbito do Programa de Formação de Pesquisas Locais.

\section{LITERATURA CITADA}

BLOUIN, M.; BAROT, S. \& LAVELLE, P. Earthworms (Millsonia anomala, Megascolecidae) do not increase rice growth through enhanced nitrogen mineralization. Soil Biol. Biochem., 38:2063-2068, 2006.

BOSSUYT, H.; SIX, J. \& HENDRIX, P.F. Protection of soil carbon by microaggregates within earthworm casts. Soil Biol. Biochem., 37:251-258, 2005.

BRITO-VEGA, H. \& ESPINOSA-VICTORIA, D. Bacterial diversity in the digestive tract of earthworms (Oligochaeta). J. Biol. Sci., 9:192-199, 2009.

BROWN, G.G.; BAROIS, I. \& LAVELLE, P. Regulation of soil organic matter dynamics and microbial activity in the drilosphere and the role of interactions with other edaphic functional domains. Eur. J. Soil Biol., 36:177-198, 2000.

BUCK, C.; LANGMAACK, M. \& SCHRADER, S. Nutrient content of earthworm casts influenced by different mulch types. Eur. J. Soil Biol., 35:23-30, 1999.

CHAOUI, H.I.; LARRY, M.Z. \& OHNO, T. Effects of earthworms casts and compost on soil microbial activity and plant nutrient availability. Soil Biol. Biochem., 35:295302. 2003 
CHAPUIS-LARDY, L.; RAMIANDRISOA, R.S.; RANDRIAMANANTSOA, L.; MOREL, C.; RABEHARISOA, L. \& BLANCHART, E. Modification of $\mathrm{P}$ availability by endogeic earthworms (Glossoscolecidae) in Ferralsols of the Malagasy Highlands. Biol. Fert. Soils, 45:415-422, 2009.

DECHEN, A.R. \& NATCHTIGALL, G.R. Elementos requeridos à nutrição de plantas. In: NOVAIS, R.F.; ALVAREZ V., V.H.; BARROS, N.F.; FONTES, R.L.F.; CANTARUTTI, B. \& NEVES, J.C.L., eds. Fertilidade do solo. Viçosa, MG, Sociedade Brasileira de Ciência do Solo, 2007. p.91-132.

EMPRESA BRASILEIRA DE PESQUISA AGROPECUÁRIA EMBRAPA. Centro Nacional de Pesquisa do Solo. Manual de métodos de análise de solo. 2.ed. Rio de Janeiro, 1997. $212 p$.

EMPRESA BRASILEIRA DE PESQUISA AGROPECUÁRIA . EMBRAPA. Centro Nacional de Pesquisa do Solo. Sistema brasileiro de classificação de solos. 2.ed. Rio de Janeiro, 2006. 306p.

FIUZA, S.S. Ecologia de Chibui bari (Annelida:Oligochaeta) e atributos físicos, químicos e biológicos de seus coprólitos. Rio Branco, Universidade Federal do Acre, 2009. 113p. (Tese de Mestrado)

GUERRA, R.A.T. Ecologia dos oligochaeta da Amazônia: 2 Estudo da estivação e da atividade de Chibui bari através da produção de excrementos. Acta Amaz., 18:27-34. 1988a.

KUSDRA, J.F.; MOREIRA, D.F.; SILVA, S.S.; ARAÚJO NETO, S.E. \& SILVA, R.G. Uso de coprólitos de minhoca na produção de mudas de mamoeiro. R. Bras. Frutic., 30:492497, 2008.

HENDRIX, P.F. Earthworms in agroecossystems in North America. Boca Raton, Lewis Publishers, 1995.

JAMES, S.W. \& BROWN, G.G. Ecologia e diversidade de minhocas no Brasil. In: MOREIRA, F.M.S.; SIQUEIRA, J.O. \& BRUSSAARD, L., eds. Biodiversidade do solo em ecossistemas brasileiros. Lavras, Universidade Federal de Lavras, 2008. p.192-276.

JONES, C.G.; LAWTON, J.H. \& SHACHAK, M. Organisms as ecosystem engineers. Oikos, 69:373-386, 1994.

JOUQUET, P.; DAUBER, J.; LAGERLOF, J.; LAVELLE, P. \& LEPAGE, M. Soil invertebrates as ecosystem engineers: Intended and accidental effects on soil and feedback loops. Appl. Soil Ecol., 32:153-164, 2006.

LAFONT, A. Effects of the earthworm Pontoscolex corethrurus on banana plants infected or not with the plant-parasitic nematode Radopholus similis. Pedobiologia, 51:311-318, 2007.

LAVELLE, P.; PASHANASI, B.; CHARPENTIER, F.; GILOT, C.; ROSSI, J. P.; DEROUARD, L.; ANDRE, J.; PONGE, J. F. \& BERNIER, N. Effects of earthworms on soil organic matter and nutrient dynamics at a landscape scale over decades. In: EDWARDS, C.A., ed. Earthworm ecology. 2.ed. Boca Raton, CRC Press, 2004. p.145-160.

LAVELLE, P.; DECAËNS, T.; AUBERT, M.; BAROT, S.; BLOUIN, M.; BUREAU, F.; MARGERIE, P.; MORA, P. \& ROSSI, J.P. Soil invertebrates and ecosystem services. Soil Biol., 42:3-15, 2006

LEE, K.E. Earthworms: Their ecology and relationships with soils and land use. Sydney, Academic Press, 1985. 411p.
LONGSDON, S.D. \& LINDEN, D.R. Interactions of earthworms with soil physical conditions influencing plant growth. Soil Sci., 154:330-337, 1992.

MALAVOLTA, E. Manual de nutrição mineral de plantas. São Paulo, Ceres, 2006. 631p.

OYEDELE, D.J.; SCHJONNING, P. \& AMUSAN, A.A Physicochemical properties of earthworm casts and uningested parent soil from selected sites in southwestern Nigeria. Ecol. Eng., 28:106-113, 2006.

PASHANASI, B.; LAVELlE, P.; ALEGRE, J. \& CHARPENTIER, F. Effect of the endogeic earthworm Pontoscolex corethrurus on soil chemical characteristics and plant growth in a low-input tropical agroecosystem. Soil Biol. Biochem., 28:801-810, 1996.

PAVINATO, P.S. \& ROSOLEM, C.A. Disponibilidade de nutrientes no solo. Decomposição e liberação de compostos orgânicos de resíduos vegetais. R. Bras. Ci. Solo, 32:911920,2008

PULLEMAN, M.M. \& MARINISSEN, J.C.Y. Physical protection of mineralizable $\mathrm{C}$ in aggregates from longterm pasture and arable soil. Geoderma, 120:273-282, 2004 .

QUADROS, R.M.B.; BELLOTE, A.F.J. \& DIONÍSIO, J.A. Observações sobre as propriedades químicas do solo e de excrementos de minhocas em plantios de Eucalyptus grandis. Bol. Pesq., 45:29-39, 2002.

ROSSI, J.P.; HUERTA, E.; FRAGOSO, C. \& LAVELLE, P. Soil properties inside earthworm patches and gaps in a tropical grassland. Eur. J. Soil Biol., 42:284-288, 2006.

SILVA, E.E.; AZEVEDO, P.H.S. \& DE-POLLI, H. Determinação da respiração basal (RBS) e quociente metabólico do solo $\left(\mathrm{qCO}_{2}\right)$. Seropédica, Embrapa Agrobiologia, 2007a. (Comunicado Técnico, 99)

SILVA, S.S.; ARAÚJO NETO, S.E.; KUSDRA, J.F. \& FERREIRA, R.L.F. Produção orgânica de mudas de couvemanteiga em substratos à base de coprólitos de minhocas. Caatinga, 20:78-83, 2007b.

SOUZA, S.R.; FONTINELE, Y.; SALDANHA, C.S.; ARAÚJO NETO, S.E. \& KUSDRA, J.F. Produção de mudas de alface com uso de substrato preparado com coprólitos de minhoca. Ci. Agrotec, 32:115-121, 2008.

SOUZA, E.D.; COSTA, S.E.V.G.A.; LIMA, C.V.S.; ANGHINONI, I.; MEURER, E.J. \& CARVALHO, P.C.F. Carbono orgânico e fósforo microbiano em sistema de integração agricultura-pecuária submetido a diferentes intensidades de pastejo em plantio direto. R. Bras. Ci. Solo, 32:1273$1282,2008$.

ZAIA, F.C.; GAMA-RODRIGUES, A.C.; GAMA-RODRIGUES, E.F. \& MACHADO, R.C.R. Fósforo orgânico em solos sob agrossistemas de cacau. R. Bras. Ci. Solo, 32:1987-1995, 2008a.

ZAIA, F.C.; GAMA-RODRIGUES, A.C. \& GAMA-RODRIGUES, E.F. Formas de fósforo no solo sob leguminosas florestais, floresta secundária e pastagem no Norte-Fluminense. R. Bras. Ci. Solo, 32:1191-1197, 2008b. 